\title{
Implications of cosmological gamma-ray absorption
}

\section{Evolution of the metagalactic radiation field}

\author{
T. M. Kneiske ${ }^{1}$, K. Mannheim ${ }^{1}$, and D. H. Hartmann ${ }^{2}$ \\ 1 Universität Würzburg, Am Hubland, 97057 Würzburg, Germany \\ 2 Clemson University, Clemson, SC 29634-0978, USA
}

Received 9 July 2001 / Accepted 30 January 2002

\begin{abstract}
Gamma-ray absorption due to $\gamma \gamma$-pair creation on cosmological scales depends on the line-of-sight integral of the evolving density of low-energy photons in the Universe, i.e. on the history of the diffuse, isotropic radiation field. Here we present and discuss a semi-empirical model for this metagalactic radiation field based on stellar light produced and reprocessed in evolving galaxies. With a minimum of parameters and assumptions, the present-day background intensity is obtained from the far-IR to the ultraviolet band. Predicted model intensities are independent of cosmological parameters, since we require that the comoving emissivity, as a function of redshift, agrees with observed values obtained from deep galaxy surveys. The far-infrared background at present predicted from optical galaxy surveys falls short in explaining the observed one, and we show that this deficit can be removed by taking into account (ultra)luminous infrared galaxies with a seperate star formation rate. The accuracy and reliability of the model, out to redshifts of $z \sim 5$, allow a realistic estimate of the attenuation length of $\mathrm{GeV}$-to-TeV gamma-rays and its uncertainty, which will be the focus of a subsequent paper.
\end{abstract}

Key words. galaxies: evolution - ISM: general - radiation mechanisms: general - cosmology: observations

\section{Introduction}

Understanding the evolution of large-scale structure in the Universe is a major goal of modern observational cosmology. Numerical simulations of hierarchical structure formation in a globally homogeneous universe are now tractable (e.g., Nagamine et al. 2000; Kauffmann et al. 1999), but connecting the evolving structures to observable fluxes of electromagnetic radiation involves uncertain empirical descriptions of star formation, supernova feedback, and the dust-gas interplay. The necessary input comes from extensive observational campaigns, such as deep galaxy surveys, which measure the number of galaxies, their morphological types, colors, fluxes, and distances in presumably representative solid angles out to redshifts of $z \sim 6$. The wealth of detailed information derived from these observations can significantly complicate the effort to link theories of galaxy evolution and large scale structure formation. It is helpful to single out global quantities for which predictions can be compared with observations. One such quantity is the cosmic star formation rate (SFR) and its associated metagalactic

Send offprint requests to: T. M. Kneiske,

e-mail: kneiske@astro.uni-wuerzburg.de radiation field (MRF) (the MRF at $z=0$ is commonly referred to as Extragalactic Background Light, EBL). The contribution of galaxies to the MRF is most significant between the far-infrared and the ultraviolet, while at longer wavelengths cosmic $2.7 \mathrm{~K}$ microwave background (CMB) radiation from the big bang dominates. At shorter wavelengths, accretion-powered active galactic nuclei provide much of the high-energy background (e.g., Mushotzky et al. 2000; Sreekumar 2000). In the GeV regime the MRF seems to originate from unresolved blazars and galaxies. Gamma rays emitted by novae, supernovae, and $\gamma$-ray bursts contribute the bulk of the observed background in the window around $1 \mathrm{MeV}$ (e.g., Watanabe et al. 1999; Weidenspointner 1999; Ruiz-Lapuente et al. 2000). In principle, the evolution of the MRF should be predictable with structure formation models (e.g., Sommerville \& Primack 1999), so that the observed MRF could be used to infer either the role of AGNs, low surface brightness objects, decays of relic particles, or to single out cosmological parameters. However, these models still rely on a wealth of uncertain parameters, and we are far from the ultimate goal of a first principles theory of the MRF. For an overview sie Hauser \& Dwek (2001) and references therein. 
Three basic methods are commonly employed for computations of the MRF from luminosity functions undergoing (i) forward evolution from a theoretically determined initial state, (ii) backward evolution from an observationally given final (present-day) state, or (iii) evolution that is directly observed over some range in redshift.

Method (i) starts from the theoretical framework of structure formation and evolution and predicts how luminosity functions evolve forward in cosmic time. The semi-analytic models of galaxy formation (e.g., White \& Frenk 1991; Baugh et al. 1998; Kauffmann et al. 1993; Sommerville \& Primack 1999; Granato et al. 2000) are based on structure formation studies with dissipationless $N$-body simulations. These studies yield luminosity functions for different morphological galaxy types that are in reasonable agreement with the observations. However, regarding the MRF, predictions from these models generally fail to satisfactorily reproduce observed cosmic emissivities. To better match these emissivities the models often require significant adjustments in the prescriptions of star formation, supernova feedback, and the inclusion of further astrophysical effects which presently can not be calculated from first principles. A simplified model for the infrared and sub-mm range was developed by Guiderdoni et al. (1998), while Malkan \& Stecker (1998, 2001), en route of method (ii), determine the infrared MRF from local luminosity functions obtained with IRAS. In a very substantial paper, Franceschini et al. (2001) employed recent ISO data and more detailed models for the IR emission.

Method (iii) computes the MRF directly from the global SFR inferred from tracers of cosmic chemical evolution, such as the various Lyman $\alpha$ systems (Salamon \& Stecker 1998; Pei et al. 1998), or from deep galaxy surveys (e.g., Madau et al. 1998; Rowan-Robinson 2001; Franceschini 2000). The spectral energy distribution (SED) for the globally averaged stellar population residing in galaxies can be estimated with population synthesis models (e.g. Bruzual \& Charlot 1993) available for various input parameters, of which the initial mass function (IMF) and metallicity are the most important ones. Reprocessing by gas and dust can be taken into account explicitly via some model of the evolution of the dust and gas content in galaxies, in combination with assumed dust properties derived from local observations in the Milky Way.

Observational attempts to determine or constrain the present-day background face severe problems due to emissions from the Galaxy, which can introduce large systematic errors. Nevertheless, a number of studies with COBE FIRAS (Fixsen et al. 1998) and COBE DIRBE (Hauser et al. 1998) have resulted in highly significant detections of a residual diffuse IR background, providing an upper bound on the MRF in the IR regime. Similarly, the cumulative flux from galaxies detected in deep HST or ISO exposures provide lower limits to the present-day MRF. In the UV, measurements of the proximity effect provide an estimate of the MRF at high redshifts (e.g., Giallongo et al. 1996). To constrain cosmic evolution of the MRF one can also utilize the fact that high-energy gamma rays (from blazars or gamma-ray bursts) originating at large redshifts are attenuated by pair creation from interactions with low-energy MRF photons (e.g. Stanev \& Franceschini 1998; Renault et al. 2001), which is the subject of a subsequent publication in this series.

Here we discuss a model of the evolving MRF that is based directly on observed emissivities (method iii), and is designed to use a minimal set of assumptions to clearly reveal the connections between input physics and output MRF. The method employed here (described in Sect. 2) is similar to the method discussed by Madau et al. (1998) or Malkan \& Stecker (1998), but we specifically address redshift evolution of the MRF and the effects of dust-reemission in the infrared, the initial mass function (IMF) and metallicity. In Sect. 3 we discuss the use of population synthesis models to relate the SFR to the observed emissivities, and describe models of the dusty ISM in star forming regions that allow us to reproduce the far-infrared bump in the present-day MRF spectrum. Despite the complexity of the underlying physics involved in the production of the MRF, one can successfully model the MRF with simple modules. This approach allows us to investigate with clarity the various factors contributing to the MRF. In Sect. 4 we present the MRF spectrum as a function of redshift and discuss in detail the dependencies on cosmological models. We discuss the effect on the IR peak induced by varying assumptions about the IMF, the mean metallicity of the emitting stars and the effect by adding a new dusty population of galaxies, ULIGs/LIGs (ultraluminous/luminous infrared galaxies). Note that the MRF determined in this way does not depend on the parameters of the assumed cosmological model. However, when we refer to comoving emissivities or the cosmic star formation rate $\operatorname{SFR}(z)$, we adopt the flat Friedmann model with $\Omega_{0}=1$, $\Omega_{\Lambda}=0$, and $h=0.5$ where $h=H_{0} /\left(100 \mathrm{~km} \mathrm{~s}^{-1} \mathrm{Mpc}^{-1}\right)$. This choice of parameters is most commonly made in the observational literature, so we employ it here to allow direct comparisons.

\section{Method}

The method for calculating the MRF from a given SFR relies on an accurate knowledge of evolving stellar spectra and the reprocessing of star light in various dusty environments. Luminosity evolution of stellar populations is sensitive to the initial mass function (IMF), evolution of the mean cosmic metallicity, and the amount of interstellar extinction. Starting point of any model is the spectral energy density (SED) produced by a population of stars resulting from an instantaneous burst of star formation (commonly normalized to the mass of stars formed). Because star formation is an ongoing process with relatively short time scales of $10^{5-7}$ yrs, the starburst spectra can be directly convolved with the global SFR, $\dot{\rho}_{*}(z)$, to derive the evolution of the global luminosity density due to cosmic star formation. The SEDs are constructed from realistic stellar evolution tracks combined with detailed 
atmospheric models (e.g., Bruzual \& Charlot 1993). The temporal evolution of the specific luminosity, $L_{\nu}(t)$ (in units of erg s ${ }^{-1} \mathrm{~Hz}^{-1}$ per unit mass of stars formed) is then determined by the choices of IMF and the initial stellar metallicity. Figure 1 shows the results for a Salpeter IMF between 0.1 and $100 M_{\odot}$ and solar metallicity. Note that the figure shows $L_{\lambda}$ as a function of wavelength. The luminosity drops rapidly as massive stars become supernovae (whose light is not included in these SEDs), and the wavelength of the bulk of the emission shifts to the red as the population ages. SEDs shown in Fig. 1 are unobscured by circumstellar gas and dust. The effects of absorption are discussed in Sect. 3.2.

From the population synthesis starburst models we obtain the comoving emissivity (or luminosity density) at cosmic epoch $t$ from the convolution

$\mathcal{E}_{\nu}(t)=\int_{t_{\mathrm{m}}}^{t} L_{\nu}\left(t-t^{\prime}\right) \dot{\tilde{\rho}}_{*}\left(t^{\prime}\right) \mathrm{d} t^{\prime}\left(\operatorname{erg~s}^{-1} \mathrm{~Hz}^{-1} \mathrm{Mpc}^{-1}\right)$

where $\dot{\tilde{\rho}}_{*}(t)=\dot{\rho}_{*}(z)$ is the star formation rate per comoving unit volume. Rewriting Eq. (1) in terms of redshift, $z=z(t)$, yields

$\mathcal{E}_{\nu}(z)=\int_{z}^{z_{\mathrm{m}}} L_{\nu}\left(t(z)-t\left(z^{\prime}\right)\right) \dot{\rho}_{*}\left(z^{\prime}\right)\left|\frac{\mathrm{d} t^{\prime}}{\mathrm{d} z^{\prime}}\right| \mathrm{d} z^{\prime}$,

where we assumed that star formation began at some finite epoch $z_{\mathrm{m}}=z\left(t_{\mathrm{m}}\right)$. For given evolution of the emissivity a second integration over redshift yields the energy density, or, after multiplication with $c / 4 \pi$, the comoving power spectrum of the MRF

$P_{\nu}(z)=\nu I_{\nu}(z)=\nu \frac{c}{4 \pi} \int_{z}^{z_{\mathrm{m}}} \mathcal{E}_{\nu^{\prime}}\left(z^{\prime}\right)\left|\frac{\mathrm{d} t^{\prime}}{\mathrm{d} z^{\prime}}\right| \mathrm{d} z^{\prime}$,

with $\nu^{\prime}=\nu\left(1+z^{\prime}\right) /(1+z)$. Cosmological parameters enter through $\mathrm{d} t / \mathrm{d} z$, which is given by (e.g., Peebles 1993 Principles of Physical Cosmology)

$\left|\frac{\mathrm{d} t}{\mathrm{~d} z}\right|=\frac{1}{H_{0}(1+z) E(z)}$

with an "equation of state"

$E(z)^{2}=\Omega_{r}(1+z)^{4}+\Omega(1+z)^{3}+\Omega_{R}(1+z)^{2}+\Omega_{\Lambda}$.

The term proportional to $\Omega_{\mathrm{r}}$ takes into account the contribution from relativistic components (such as the CMB and the star light). The density parameter of this component is defined as $\Omega_{\mathrm{r}}=u_{\mathrm{r}} / \rho_{\text {crit }} c^{2}$, where $u_{\mathrm{r}}$ refers to the relativistic energy density and $\rho_{\text {crit }}$ is the critical density of the universe; $\rho_{\text {crit }}=3 H_{0}^{2} / 8 \pi G=10.54 h^{2} \mathrm{keV} / \mathrm{cm}^{3}$. The resulting dependencies on the Hubble constant are; emissivity $\propto H_{0}^{-1}$, and MRF power spectrum $\propto H_{0}^{-2}$. However, this scaling is only correct if we formally require that the star formation rate is a given function of redshift. Lacking a reliable derivation from first principles, this function is derived from observations that involve distance and luminosity estimates, which introduces additional powers of $H_{0}$.

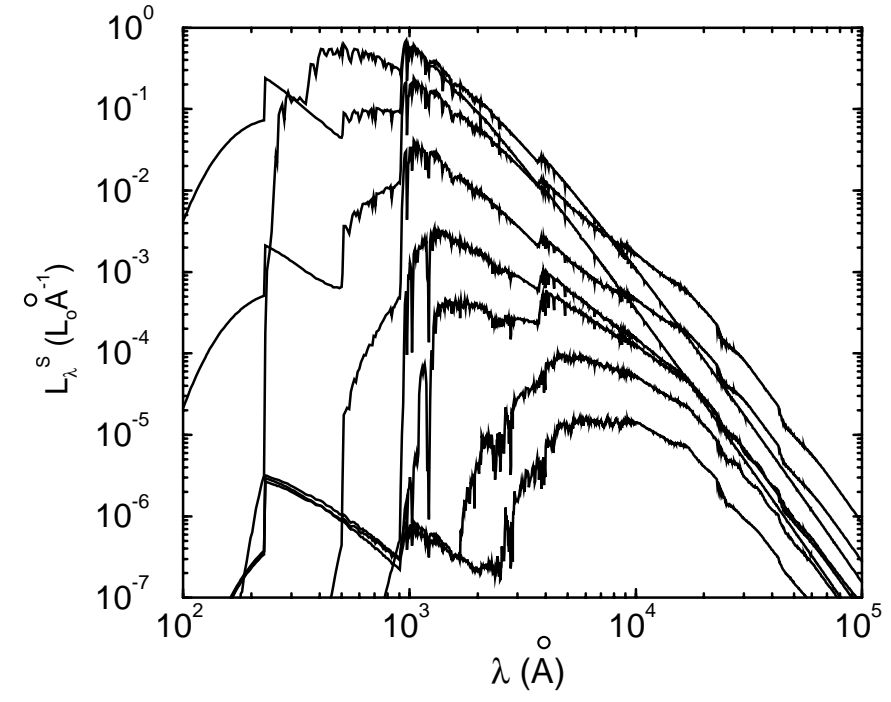

Fig. 1. Spectral energy distributions (SEDs) of a coeval stellar population as a function of age $\tau$. The specific luminosity of the evolving stellar population is normalized to one solar mass. The stellar models assume standard solar composition. Shown are the SEDs for ages $\tau$ 0, 0.0038, 0.00724, 0.0138, 0.07187, $0.28612,1.434$, and 11 Gyrs (from top to bottom, based on calculations by Bruzual \& Charlot 1999).

\section{Emissivity}

\subsection{Spectral synthesis model}

Conversion of gas to stars produces a stellar mass distribution that is commonly described by a "universal" initial mass function. A batch of stars produced in an instantaneous "burst" of star formation is often referred to as a Simple Stellar Population (SSP). Massive stars in the SSP have short lives $\left(\sim 10^{7}\right.$ yrs $)$ and predominantly produce UV radiation, while long-lived, low-mass stars remain close to the main sequence even over cosmological times and produce the bulk of the "red light". Depending on their mass, stars follow different evolutionary paths and evolve on different time scales, which causes the SED of a SSP to be a sensitive function of time. To follow the changing energy output in time we use the population synthesis code of Bruzual \& Charlot 1999 (BC-Model), which is an updated version of the code documented in GISSEL96 (Leitherer et al. 1996). Figure 1 shows the resulting SEDs emitted at several distinct times after the burst. The stellar spectra used to construct these SEDs are based on Padova tracks (e.g. Girardi et al. 2000) and Lejeune stellar atmosphere models (Lejeune et al. 1997, 1998), and include a post-AGB evolutionary phase. For demonstration purposes we adopted a population with solar metallicity and Salpeter single power law IMF in the range $0.1 M_{\odot}<M<100 M_{\odot}$, although different choices can readily be made with this code. The SEDs shown in Fig. (1) are of course not representative of any particular type of galaxy, because those involve star formation histories that are usually different from a single burst. However, the SEDs do resemble more closely the spectra of 
elliptical galaxies, for which the single star burst might be a reasonable approximation.

\subsection{Interstellar medium}

In contrast to the intergalactic medium (IGM), which is extremely tenuous and has only a small effect on the transmitted SEDs from redshifts less than unity (e.g., Madau 2000), the intrinsic absorption by the galactic interstellar medium (ISM) is significant, and must be included to obtain correct SEDs. For simplicity, we assume a uniform distribution of gas and dust surrounding the stars of the SSP, and apply Osterbrook's Case B recombination for optically thick clouds at an equilibrium temperature of $10^{4} \mathrm{~K}$, i.e. total absorption of all ionizing photons and reemission of $68 \%$ of the absorbed power in $L_{\alpha}$ line emission (which is subsequently absorbed by dust). The remaining energy is assumed to be reemitted in the optical regime via bremsstrahlung and recombination line/continuum emission. The assumption of homogeneity of the absorbing gas and dust layers is a simplification that could have a noticeable effect on the estimated transmission of UV radiation shortward of the Lyman edge. A more realistic approach should also take into account ionized superbubbles that are driven into the ISM by multiple supernovae (e.g., Dove et al. 2000).

The average metallicty of gas in galaxies slowly increases with cosmic time, but the present-day value is not known precisely (e.g., Pei et al. 1999). We thus adopt an average extinction curve

$A_{\lambda}=0.68 \cdot E(B-V) \cdot R \cdot\left(\lambda^{-1}-0.35\right)$

with $R=3.2$ and where $A_{\lambda}$ with $\lambda[\mu \mathrm{m}]$ determines the absorption coefficient according to $g(\lambda)=10^{-0.4 \cdot A_{\lambda}}$. Reemission is calculated as the sum of three modified Planck spectra

$L_{\lambda}^{\mathrm{d}}\left(L_{\mathrm{bol}}\right)=\sum_{i=1}^{3} c_{i}\left(L_{\mathrm{bol}}\right) \cdot Q_{\lambda} \cdot B_{\lambda}\left(T_{i}\right)$

where $Q_{\lambda} \propto \lambda^{-1}$ and $L_{\mathrm{bol}}=L_{\mathrm{bol}}(\tau)$.

Two temperatures characterize warm and cold dust in galaxies. The third temperature is included to model a PAH component, which is assumed to emit like a Blackbody. Emission lines are not treated separately, because of the smoothing effects from integration over redshift. We normalize these three components realtive to each other (with the constants $c_{i}, i=1 \ldots 3$ ) by using nonSeyfert galaxy observations by Spinoglio et al. (1995). We fitted the relation for all 4 bands.

Dust in the ISM of the Milky Way is known to coexist at several different temperatures, determined by the distances from various heat sources. Hot dust in spiral galaxies has temperatures ranging from $50 \mathrm{~K}$ to $150 \mathrm{~K}-200 \mathrm{~K}$ (Sauvage et al. 1997 and references therein) when in thermal equilibrium with HII regions, young massive stars, or compact accreting sources. Radiation from this dust component emerges in the mid-infrared and reprocesses only a small fraction of the emitted luminosity. Warm dust with temperatures between $25 \mathrm{~K}$ and $50 \mathrm{~K}$ corresponds to regions heated by the mean interstellar radiation field. Dust inside molecular clouds is somewhat shielded against highenergy radiation, and thus appears at low temperatures between $10 \mathrm{~K}$ and $25 \mathrm{~K}$. Very cold dust at temperatures of $10 \mathrm{~K}$, or even less, can be present in the densest parts of molecular clouds or in outer regions of the galaxy where the flux of the interstellar radiation field has dropped to the value of the intergalactic radiation field. Such very cold dust is difficult to detect, and requires sub-mm observations which so far have failed to provide unambiguous results. Therefore, we do not include very cold dust in our model. To keep the model simple, we consider variations in dust composition only because the shape of the spectrum is dependent of the total flux. It is noteworthy, however, that emission features around $10 \mu \mathrm{m}$ due to Polycyclic Aromatic Hydrogen (PAH) molecules seem to be ubiquitous in galaxies (Desert el al. 1990). These PAH's are undergoing temperature fluctuations and are generally not in thermal equilibrium. The broad emission lines of the PAHs are modeled with an additional (low flux) blackbody component, characterized by $T \sim 425 \mathrm{~K}$ (Dwek et al. 1997).

As mentioned above, we use the non-Seyfert galaxy relations for all 4 bands by Spinoglio et al. (1995) to fix the 6 model parameters in Eq. (7) (three temperatures $T_{i}$, $i=1 \ldots 3$ and three constants $\left.c_{i}, i=1 \ldots 3\right)$. The relations depend on the total luminosity which is radiated by a galaxy. Although we are using a SSP and not a "real" galaxy we can use the IR relations of galaxies, because these relations only depend on the total luminosity of a galaxy. We use $10^{11} M_{\odot}$ for each SSP spectrum to get a galaxy-like total luminosity. Note that the dust-spectra $L_{\lambda}^{\mathrm{d}}$ depend on the age $\tau$ of the SSP, because they change with total luminosity $L_{\text {bol }}$.

As starting values for the temperature we use $T_{1}=$ $240 \mathrm{~K}$ (consistent with a PAH blackbody of $425 \mathrm{~K}$ ), $T_{2}=80 \mathrm{~K}$ (warm dust) and $T_{3}=30 \mathrm{~K}$ (cold dust). After the fitting procedure we obtain for each SSP spectrum the associated IR-spectrum (see Fig. 2). The resulting temperatures are higher for higher total luminosities (for younger SSPs) and they are generally in the range $27 \mathrm{~K}<T_{1}<33 \mathrm{~K}, 125 \mathrm{~K}<T_{2}<70 \mathrm{~K}$, and $180 \mathrm{~K}<T_{3}<400 \mathrm{~K}$.

The resulting total spectra, including absorption and reemission, can be written for each SSP-spectrum as

$L_{\lambda}(\tau)=\left[\left(L_{\lambda, \mathrm{BC}}(\tau)+c_{\mathrm{g}}(\tau) \cdot \epsilon_{\lambda}\right)\right] \cdot g_{\lambda}+c_{\mathrm{d}} \cdot L_{\lambda}^{\mathrm{d}}(\tau)$

where $L_{\lambda, \mathrm{BC}}(\tau)$ are the SEDs from the BC-Model with an age $\tau$. The optical emission of gas heated by absorbed photons is included through the quantity $\epsilon_{\lambda} \propto \exp (-h \nu / k T)$ multiplied by the fractional energy $c_{\mathrm{g}}$ available for this channel $(32 \%)$. Both spectral components are modified by the absorption coefficient $g_{\lambda}$. The dust reemission spectra $L_{\lambda}^{\mathrm{d}}(\tau)$ are added according to Eq. (7). The IR-spectra shown in Fig. 2 are normalized with $c_{\mathrm{d}}$ using energy conservation of the absorbed and re-emitted photons. 


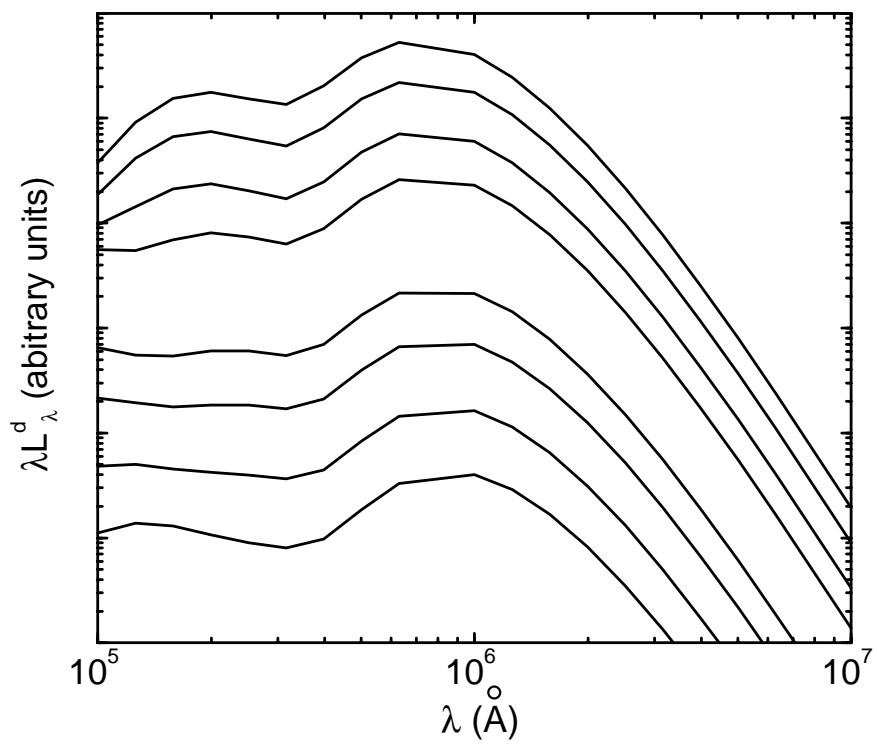

Fig. 2. IR-spectra associated with each of the SSP-spectra shown in Fig. 1. The IR emission is modeled as the sum of three modified blackbody spectra. The determination of the temperatures of these components is discussed in the text.

Published values for the color index, $E(B-V)$, cover a wide range, which reflects large uncertainties associated with dust properties. Steidel et al. (1999) adopt $E(B-V)=0.15$ at redshifts $z=3-4$. Madau et al. (1998) use a universal value of $E(B-V)=0.1$, Guiderdoni (1999) prefers $E(B-V)=0.09$ at $z>2$. Generally, dust extinction seems to play a more important role at high redshifts (e.g., Pettini et al. 1998). According to Madau et al. (1998) the index varies as $E(B-V)=0.011(1+z)^{2.2}$.

Using the relation $\lambda L_{\lambda}(\tau)=\nu L_{\nu}(\tau)$ we calculate $L_{\nu}$ to obtain the emissivity in Eq. (2).

\subsection{Star formation history}

Recent deep galaxy surveys (e.g., Kennicutt 1983, 1999) or Lyman $\alpha$ absorber studies (Pei \& Fall 1995; Pei et al. $1999)$ suggest a functional shape of the $\operatorname{SFR}\left(\dot{\rho}_{*}(z)\right)$ that can be approximated with a simple broken power law

$\dot{\rho_{*}}(z) \propto(1+z)^{\alpha}$

with $\alpha=\alpha_{\mathrm{m}}>0$ for $z \leq z_{\text {peak }}$ and $\alpha=\beta_{\mathrm{m}}<0$ for $z>z_{\text {peak }}$. Plotted as a function of redshift Fig. 3 (bottom) shows the fit function in comparison to the more complex fit function given in Madau (1999). The cosmic star formation rate density $\operatorname{SFR}(z)$ has been determined with different methods and for large set of input data, as recently summarized by Ruiz-Lapuente et al. (2000). These studies suggest that the original Madau curve, Madau (1997(II)), should be considered a lower limit, and that realistic rates could be larger by a factor $2-3$ at all redshifts. The compilation of Ruiz-Lapuente et al. (2000) (see their Fig. 1) clearly shows that we do not yet understand systematic effects well enough to
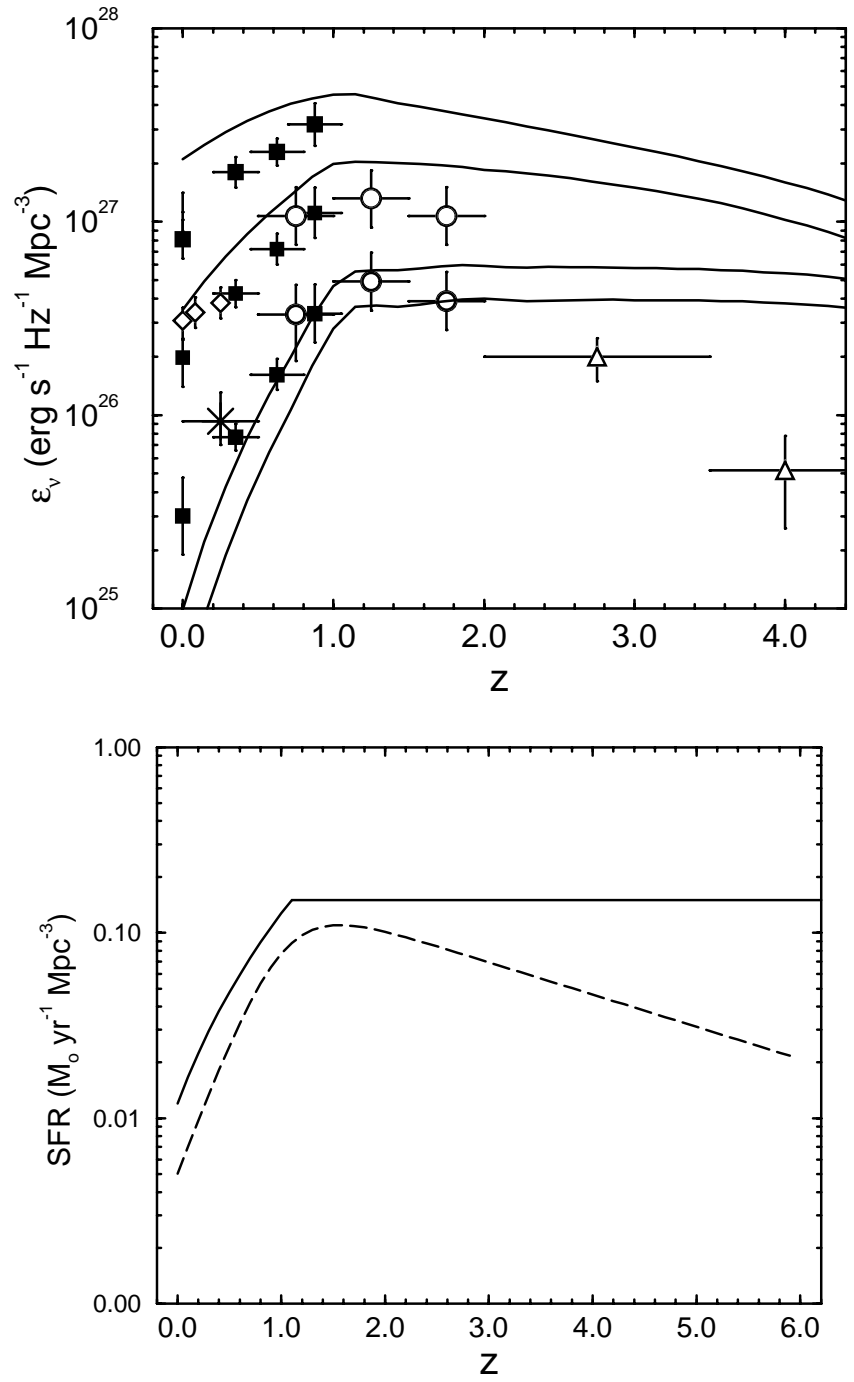

Fig. 3. a) Redshift dependence of the emissivity corresponding to the star formation history shown in the bottom panel. Solid lines show model emissivities at $1.0 \mu \mathrm{m}, 0.44 \mu \mathrm{m}, 0.28 \mu \mathrm{m}$ and $0.16 \mu \mathrm{m}$ from top to bottom. Data plotted with solid squares are taken from Lilly et al. (1996), open circles: Conolly et al. (1997), solid diamonds: Ellis et al. (1996) and open triangles: Pozzetti et al. (1998; lower limits at high redshift and $0.16 \mu \mathrm{m})$. b) Comoving star formation rate density as a function of redshift. The solid line is the rate used for computing the emissivity (with $\alpha=3.4, \beta=0, z_{\text {peak }}=1.1$, $\left.\dot{\rho}_{*}\left(z_{\text {peak }}\right)=0.15 M_{\odot} \mathrm{yr}^{-1} \mathrm{Mpc}^{-3}\right)$. The dashed line is a fit function provided by Madau (1999).

obtain a reliable estimate for $\operatorname{SFR}(z)$. This is especially true at redshifts much beyond unity.

In our approach to modeling the MRF, the SFR function is considered to be a free fit function aimed at reproducing the emissivities derived from deep surveys. "Measurements" of the SFR are generally based on luminosity densities, and thus model dependent. It is thus preferable to use the emissivities directly to obtain a selfconsistent star formation history. We note, that the SFR parameterization used here does not contain any cosmological parameters. However, it is clear that choosing a 
different cosmology does change the observationally determined emissivities (Lilly et al. 1996; Ellis et al. 1996), hence requiring a different SFR.

For a given SFR, the emissivity is readily obtained from the convolution given by Eq. (2). The resulting $\mathcal{E}_{\nu}(z)$ is plotted in Fig. 3 for four different wavelengths in the optical band, and compared to the observations. Note that the steep increase at $0.28 \mu \mathrm{m}$ and the shallower increase at $1.0 \mu \mathrm{m}$ are reproduced by the model. The model slightly overproduces the present-day emissivity at $1.0 \mu \mathrm{m}$. However, the data point of Lilly et al. (1996) at $0.44 \mu \mathrm{m}$ and $z=0$ falls much below the corresponding value obtained by Ellis et al. (1996), indicating the conservative nature of the Lilly et al. measurements. Generally, there is good agreement with the data.

\subsection{Contribution from luminous infrared galaxies}

Luminous Infrared Galaxies (LIGs) were first discovered with IRAS (Soifer et al. 1987). They represent a population of galaxies with IR luminosites above $10^{11} L_{\odot}\left(L>10^{12} L_{\odot}\right.$ are named ultraluminous infrared galaxies ULIGs) and high star formation rates. Most of these galaxies are dust enshrouded starburst galaxies or mergers, some of the ULIGs have also been identified as AGN (e.g. Kim et al. 1998). Although the LIGs are not so numerous today, a significant fraction of the infrared light could originate from them. Moreover, their star formation history could well be different from the SFR of optical galaxies (Franceschini et al. 2001). As these galaxies do hardly show up in optical surveys $\left(E(B-V)_{\text {LIG }}>\right.$ $\left.E(B-V)_{\mathrm{OPT}}\right)$, there existence is not reflected in the SFR discussed in the previous section. Also, the dust temperatures in LIGs are higher, hence we emphasize that adding another $\mathrm{SFR}_{\mathrm{LIG}}$ is a straightforward ramification of the model which would affect the infrared part of the MRF. In the following we will start to model the MRF without this contribution, but we will return to it later.

\section{Results}

\subsection{Metagalactic radiation field}

The final step in computing the MRF involves an integration of the emissivity over cosmic time using Eq. (3), where we neglected spectral modifications due to the intergalactic medium (IGM). The IGM consists predominantly of Lyman- $\alpha$ clouds with HI, HII and HeI gas (e.g., Madau 1997(I)) and mainly affects photons with wavelengths shortward of $911 \AA$ which we assumed to be completely absorbed inside galaxies. Recent work by Pei et al. (1999) also shows that absorption in the IGM, if present, is minimal. The neglect of IGM effects can cause a slight underestimate of the star formation rate, but will not affect the estimate of the MRF spectrum, because our fit procedure uses the observed emissivities, which guarantees that all photons contributing to the MRF are accounted for. The evolution of the comoving MRF spectrum is shown
Table 1. Integrated diffuse background for different models.

\begin{tabular}{|c|c|}
\hline & $I(0)\left[\mathrm{nW} \mathrm{m}^{-2} \mathrm{sr}^{-1}\right]$ \\
\hline This Model & $46^{1}$ \\
\hline Range from Data & $55 \pm 20^{2}$ \\
\hline Dwek et al. (1998) (UVO) ${ }^{3}$ & 30 \\
\hline (PFI) $)^{4}$ & 91 \\
\hline (PFC) $)^{5}$ & 41 \\
\hline Pei et al. (1998) & $51-55$ \\
\hline
\end{tabular}

in Fig. 4 for several redshifts, where the wavelength scale corresponds to a comoving reference frame.

\subsection{Dependence on cosmology}

Formally, the MRF intensity computed according to the procedures outlined in Sect. 2 exhibits an explicit cosmology dependence. This is the consequence of choosing an apparently cosmology-independent SFR. Such a choice is unrealistic, because if one were to change the cosmological model, while keeping the same SFR, one would immediately fail to reproduce the observed emissivities. However, the emissivities themselves are computed directly from observables, viz. the fluxes $\mathcal{F}$ and redshifts of galaxies. We therefore have to take a look at the cosmology dependence of the emissivities implied by the relation

$\mathcal{E}(z)=\frac{\mathrm{d} L}{\mathrm{~d} V_{\mathrm{c}}}=\frac{4 \pi d_{\mathrm{L}}^{2}(z) \mathrm{d} \mathcal{F}}{\mathrm{d} V_{\mathrm{c}}}$

where $d_{\mathrm{L}}(z)$ is the luminosity distance and

$\mathrm{d} V_{\mathrm{c}}=\frac{d_{L}^{2} \mathrm{~d} \Omega \mathrm{d} z}{H_{0}(1+z) E(z)}$

the comoving volume element, and hence $\mathcal{E}(z) \propto H_{0}(1+$ $z) E(z)$. Inserting this in Eq. (3) strictly cancels the cosmology dependence. This argument means that by requiring the model to fit measured emissivities (evaluated for a given cosmology) the resulting MRF no longer explicitely depends on the choice of cosmological parameters. The background radiation field becomes, in a sense, a measured quantity itself, since both luminosity and volume scale $\propto d_{1}^{2}$.

\subsection{Bolometric flux}

We complete this section with a comparison of the "bolometric" (IR - opt) flux obtained from our model with results available in the literature (Table 1). A strict lower limit on the present-day MRF flux of $28 \mathrm{nW} \mathrm{m} \mathrm{m}^{-2} \mathrm{sr}^{-1}$ was derived from COBE and HST data (Dwek et al. 1998). The integrated flux from our model, $46 \mathrm{nW} \mathrm{m}^{-2} \mathrm{sr}^{-1}$, is in agreement with all models using a similar SFR.

\footnotetext{
${ }^{1} I(0.2)=45 ; I(0.4)=44 ; I(0.6)=42 ; I(1.0)=35 ; I(2.0)=$ $15 ; I(3.0)=7 ; I(4.0)=2$.

2 Pozzetti \& Madau (2000).

3 Using SFR-Madau et al. (1998).

4 Using SFR-infall model (Pei \& Fall 1995).

5 Using SFR-closed box model (Pei \& Fall 1995).
} 


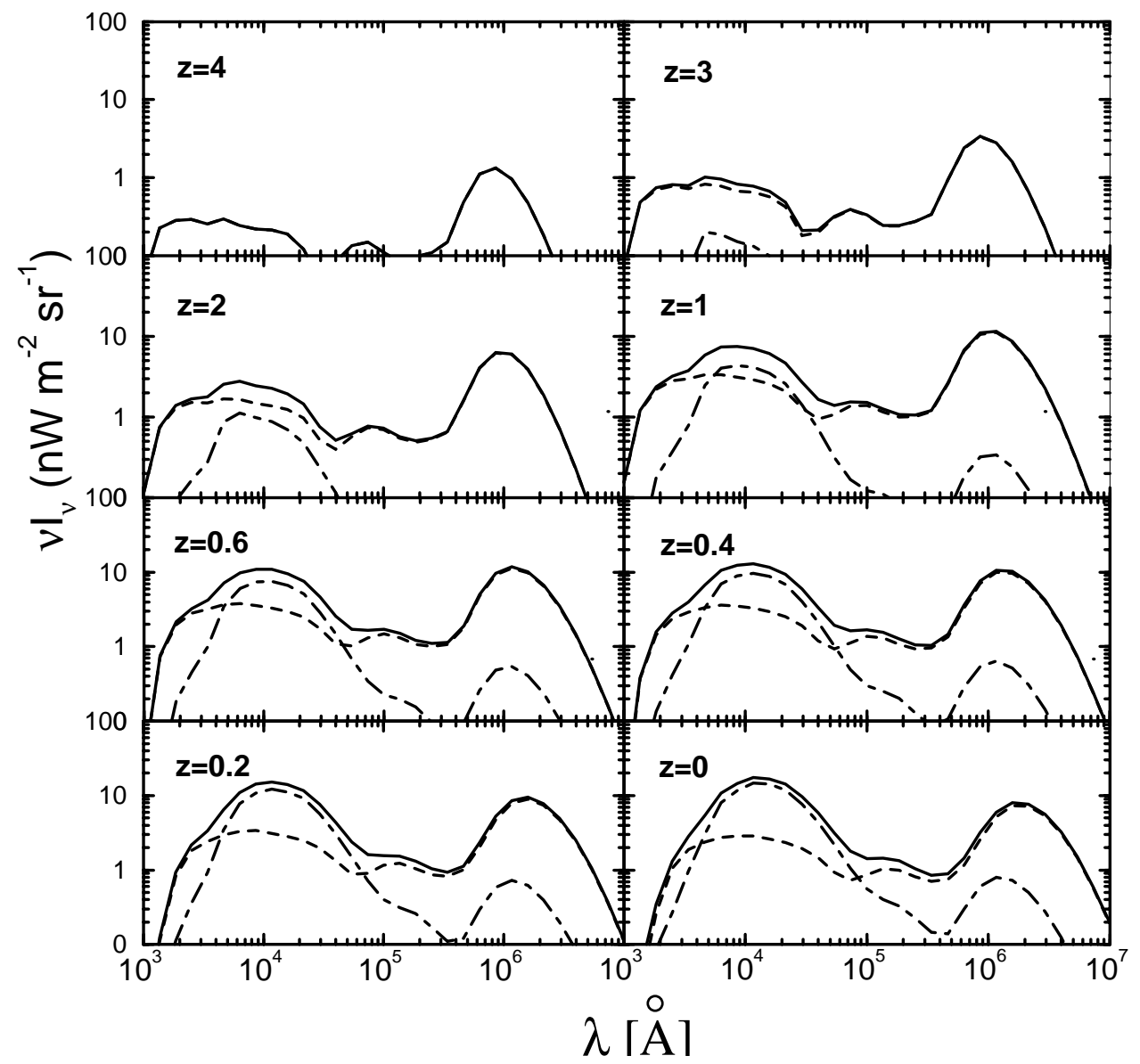

Fig. 4. The evolving spectrum of the extragalactic background light (with model parameters as in Fig. 3) as a function of wavelength (in Angstroms). The dashed lines show the contribution of massive stars (MS life <0.3 Gyr) and the dot-dashed line the contribution of low mass stars (MS life $>0.3$ Gyr).

\section{Discussion}

We have developed a simple model and its evolution with time. This model is based on direct measurements of the global emissivities due to galaxies. There are no measurements of the MRF at high redshifts that could be used to directly verify our model, with the possible exception of the UV background based on the proximity effect (e.g., Giallongo et al. 1996).

In the second paper of this series we will introduce a method to constrain the MRF at high redshift using this model and high-energy observations of blazars. For now this leaves us with various measurements of the presentday MRF as the most relevant set of constraints. While the match is by no means perfect, the comparison shown in Fig. 5 suggests that our simple model is capable, without fine-tuning of parameters, of explaining the global level and the general spectral shape of the MRF. But the predicted flux in the IR-band falls short by roughly a factor two. As discussed above, the magnitude as well as the shape of the MRF are the result of a convolution of SEDs from an aging stellar population with continuous star formation at a given rate $\operatorname{SFR}(z)$ and the cosmological effects of redshift and time dilation. The fact that our model roughly matches both magnitude and shape of the MRF indicates that the relevant input physics has been properly taken into account. This provides confidence in the predictions of the MRF at higher redshifts, which is needed for a variety of astrophysical studies. However, there are still some deviations that need to be addressed, especially the shortfall in the IR band.

\subsection{Population synthesis models}

There are several model parameters that introduce significant uncertainties in the estimated MRF flux. Most important among them are the IMF, the dust extinction model, the parameterization of the dust emission by multiple blackbodies with different temperatures, the metallicity dependence of stellar evolution tracks, and the amplitude and form of the SFR (especially at redshifts less than unity). The choice of cosmology plays only a minor role for the MRF spectrum, as we emphasized in the previous section. We also investigated different population synthesis templates for the stellar light output (e.g., Leitherer et al. 1996 and references therein) and found them to be very similar to each other. The choice of the population synthesis model thus does not significantly affect the estimated MRF. 


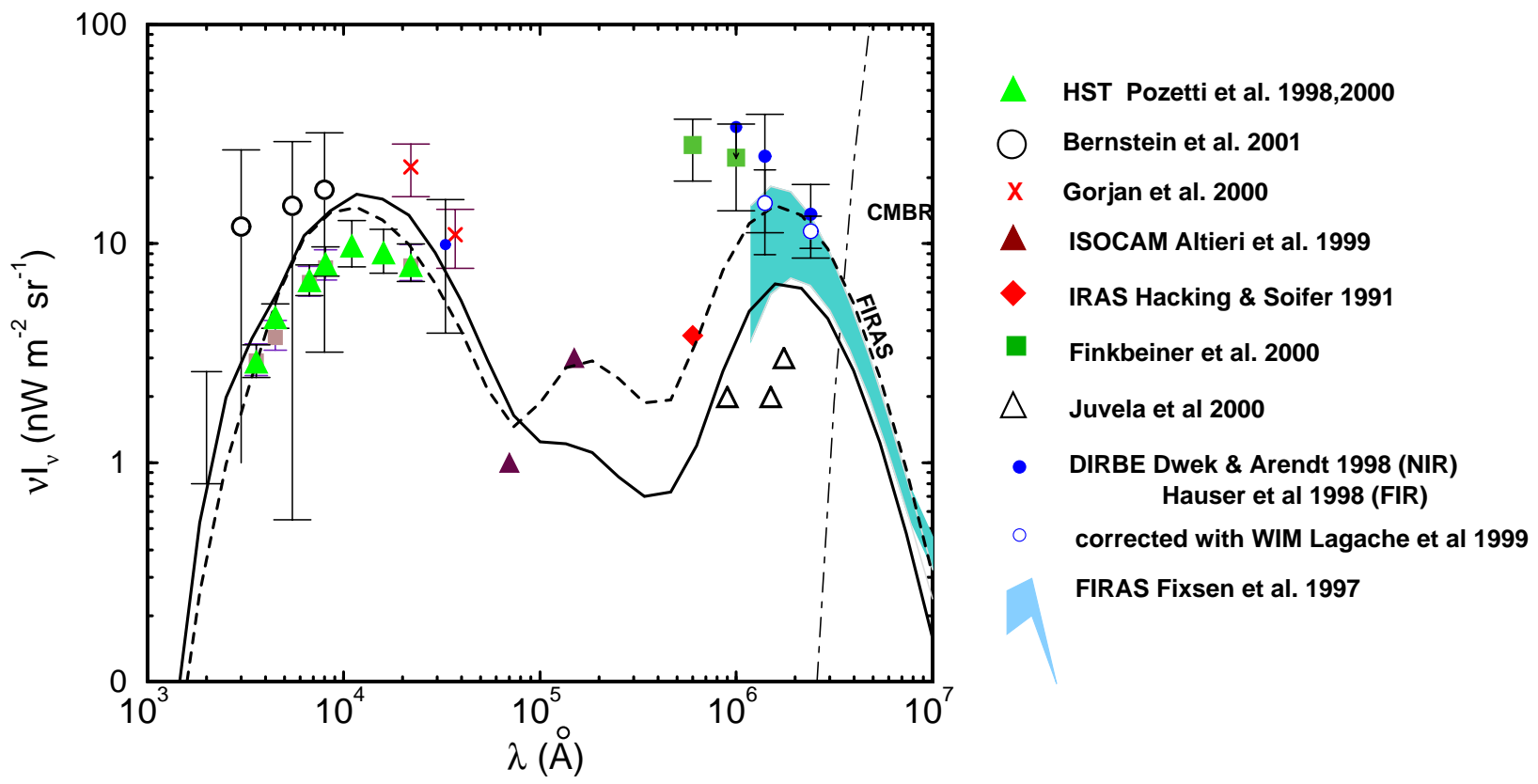

Fig. 5. The EBL computed with the model (solid line). Parameters are: $Z=0.02, E(B-V)=0.14$ (young population), $E(B-V)=0.03$ (old population), SFR-parameters see Fig. 3. The deficit in the far-infrared can be avoided using a (perhaps unrealistically) low value of the metallicity $(Z=0.0001)$. The effect of low- $Z$ stellar atmospheres is to produce relatively more UV radiation which is subsequently redistributed to the FIR by interstellar dust grains.

\subsection{IMF}

We proceed with a discussion of those parameters that, at least in principle, can significantly alter the estimated intensity, flux, or energy density. We start by comparing results based on the Salpeter and Scalo IMF (see Fig. 5). One of the distinguishing features of the Scalo IMF is the fact that it contains relatively few high-mass stars. These stars are responsible for most of the UV photons, which, after thermalization by the dust in the ISM, emerge in the FIR. On the other hand, the Scalo IMF contains a relatively large fraction of low mass stars which emit most of their light at optical wavelengths. Consequently the present-day MRF has a much weaker FIR bump when the Scalo IMF is employed. This perhaps provides an argument in favor of the Salpeter form for a global IMF, because the Scalo IMF would somewhat underproduce the FIR background.

\subsection{Star formation rate}

The star formation rate density obtained here (see Fig. 3) is higher than the SFR originally suggested by Madau (1997(II), 1999), and is somewhat different from the rate derived from structure-formation theory (Primack et al. 1998). As discussed above, recent determinations of the cosmic star formation history based on $\mathrm{H}_{\alpha}$ emission and ISO data suggest that the Madau rate has been systematically underestimated by a factor 2-3 (see Flores et al. 1999 for a recent discussion). The measurements of the sub-mm SCUBA array (Hughes et al. 1998) support the notion that much of the star formation activity at high redshifts is hidden due to dust absorption. Ruiz-Lapuente et al. (2000) summarize many of these measurements and compare (their Fig. 1) the various functions with that of Madau et al. (1998). The function used in this study falls above the "Madau curve", but below most of the curves compiled by Ruiz-Lapuente et al. (2000).

\subsection{Absorption and re-emission of the ISM}

The dust and gas model we use is not based on first principles, but is founded on empirical results. A three temperature model for galactic dust spectra has been proposed by Dwek et al. (1998). A small change in the temperatures (say, $\pm 10 \mathrm{~K}$ ) would only cause a small change in the shape of the MRF spectrum. This lack of sensitivity originates from the broadening of the employed modified blackbody spectra due to the integration over redshift.

Any modification of $E(B-V)$ changes the spectral shape of the MRF from $911 \AA$ to $10^{4} \AA$, especially the amplitude of the far-infrared bump. This is simply due to energy conservation. An increase in the extinction causes a larger fraction of UV absorption, and this energy reemerges predominantly in the FIR. We selected a value of $E(B-V)$ that provided an acceptable fit to the available data on emissivities as a function of redshift and the present-day background. We found that $E(B-V)=0.14$ is the appropriate value for young SSP's and 0.03 for old SSP. These values are reasonably well determined by the emissivity-fit alone. In any case, changing the $E(B-V)$ value does not provide a solution to the "missing IR flux". 


\subsection{Comparison with data and other models at $z=0$}

The model present-day MRF flux at optical wavelengths is consistent with lower limits from HST (Pozzetti et al. 1998, 2000). Observations by Bernstein et al. (2000) suggest the possibility of a somewhat higher MRF flux, but our results still fall within their estimated uncertainties.

Absolute measurements (albeit with large systematic errors due to the need for subtraction of dominant local foreground emission (Lagache et al. 1999; Puget \& Lagache 2000) are available from COBE/DIRBE and FIRAS in the IR band. A discrepancy between our model and the observations occurs at $\mu \mathrm{m}$ wavelengths, where the measured flux appears to be larger by a factor of two. A lower limit based on galaxy counts due to Elbaz et al. (1999) at $\sim 10^{5} \AA$ lies in the range of possible PAH emission.

While the implications for the MFR at high redshifts are rarely stated in the literature, considerable effort has gone into the calculation of the present-day MFR, i.e., the extragalactic background light (EBL). In spite of great diversity in the computational approaches employed (see the discussion in the introduction), the models generally show the same IR deficit noticed in this study (e.g. Dwek et al. 1998; Primack 1998). Dwek et al. suggested a possible solution by adding a new, distinct component of obscured galaxies which emit preferentially in the IR band.

\subsection{Metallicity}

The calculations for our "standard model" employ stellar models with solar metallicity. Salamon \& Stecker (1998) and Pei et al. (1999) discuss some of the possible effects caused by changes in the mean cosmic metallicity. The approximation of a fixed, high (solar) metallicity is motivated in part by structure formation simulations (e.g., Valageas \& Silk 1999) which suggest that the mean metallicity in star forming regions is a slowly rising function for redshifts less than 2. The calculations of Valageas \& Silk also indicate that the metallicity has been larger than $1 / 2 Z_{\odot}$ since $z \sim 2$ (note that their estimate only includes enrichment due to SNII). The significantly lower metallicity values found in damped Lyman Alpha systems (DLAs; e.g., Pettini et al. 1997) probably correspond to the enrichment history of galactic halos. The trend of $[\mathrm{Zn} / \mathrm{H}]$ with redshift suggests a present-day metallicity of $1 / 3$ solar (Vladilo et al. 2000), which also indicates that DLAs do not trace the chemical evolution of proto-disks, but instead star-forming fragments which build up galaxies through mergers. Although the Milky Way does not represent a good template for cosmic chemical evolution (e.g., Prantzos \& Silk 1989; Fields 1999) its age-metallicity relation and well known radial metallicity gradients suggest that much of its current star forming activity takes place in environments with $Z \sim Z_{\odot}$, or even higher (see Boissier \& Prantzos 1999 for a recent model of galactic chemical evolution). We thus expect the $Z=Z_{\odot}=$ const. assumption to provide a reasonable approximation. However, to test the dependence of our results on metallicity, we performed the MRF simulation for two cases: i) solar metallicity $Z_{\odot}=0.02$, and ii) $Z=0.0001$. The dashed lines in Fig. 6 show the "low- $Z$ MRF" in comparison to the standard model with solar metallicity (solid line). Both simulations used the Salpeter IMF. The comparison indicates that metallicity effects could be important. Lower metallicity in the stellar atmospheres leads to a higher fraction of light primarily emitted in the UV, and subsequently redistributed towards the IR by interstellar dust grains, with extinction parameters determined newly from the fit to the emissivities (i.e. independent of the assumed low metallicity in the stellar atmospheres producing the bulk of the light). A rigorous treatment of metallicity effects, in order to obtain self-consistent interstellar extinction curves and chemical evolution (Pei et al. 1999), is beyond the scope of the paper.

\subsection{ULIGs/LIGs}

The approach in our model up to this point was to consider the emission from galaxies found in deep optical surveys, and to compute a mean galaxy spectrum for them. This is certainly a valid scheme to obtain a lower limit to the MRF, and, in fact, the model does not overproduce observational upper limits at any frequency. However, the infrared deficit revealed by the preceding analysis, seems to justify the inclusion of a population of luminous infrared galaxies (see Sect. 3.4). This would increase the strength of the infrared bump in the MRF, but leave the optical bump unchanged. Hence the overall performance of the model to reproduce the present-day extragalactic background spectrum can be improved. The star formation history used for this population $\mathrm{SFR}_{\mathrm{LIG}}$ (Dwek et al. 1998) has been infered from number counts in ISO, IRAS and SCUBA data, and the luminosity functions from Chary \& Elbaz (2001) (and references therein). The extinction parameter is set to be $E(B-V)=1.0$, a value which is typical for LIGs. The resulting $\operatorname{SFR}_{\text {LIG }}\left(\alpha=5.5, \beta=0, z_{\text {peak }}=1.0\right.$, $\left.\dot{\rho}_{*}(z)=0.10 M_{\odot} \mathrm{yr}^{-1} \mathrm{Mpc}^{-3}\right)$ is in agreement with the one calculated by Chary \& Elbaz. At low redshifts the contribution of stars in LIGs is very small, but at high redshifts the number of stars formed in LIGs is comparable to the one in optically selected galaxies. This is in line with the interpretation of the IRAS (Kim \& Sanders 1998) observations, which indicate that LIGs were more numerous in the past than they are today. Figure 6 shows the EBL and the respective contributions from optical and infrared galaxies.

\section{Conclusions}

We have developed a model for the evolving MRF based on optical galaxy surveys as its main observational input, and found that this model shows a deficit at infrared wavelengths in the spectrum of the EBL (the MRF at $z=0$ ). Inclusion of obscured, infrared-emitting galaxies provides a viable solution of the problem, and we 


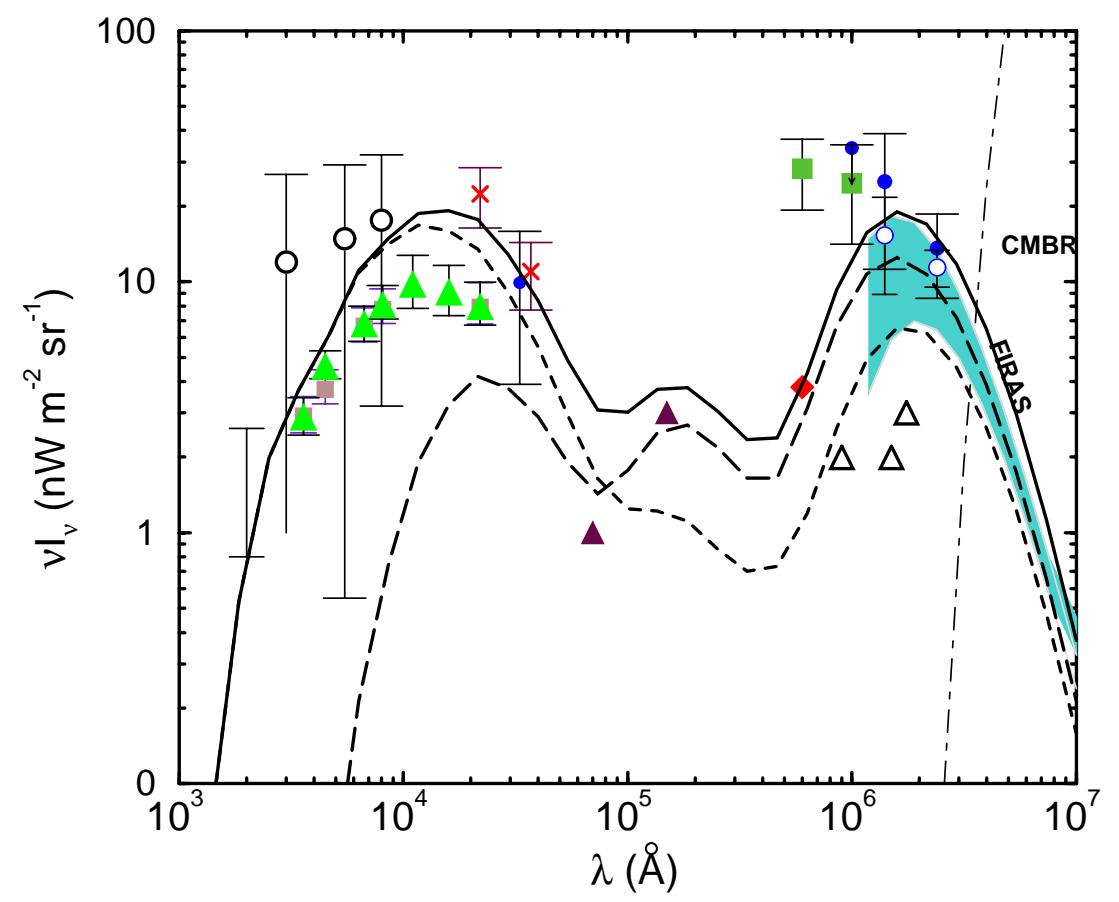

Fig. 6. The EBL computed with the model including a separate LIG population (solid line), for the same parameters that were used to produce the solid line in Fig. 5. The long dashed line shows the contribution from LIGs (parameters see text 5.7), the short dashed line the contribution from optical galaxies.

have determined their SFR from fitting the model to EBL data. The model in this form can serve as a reliable basis for obtaining predictions of the MRF at high redshifts. Observations of high-redshift gamma ray sources with next-generation gamma ray telescopes (GLAST, HESS, MAGIC, VERITAS) are expected to soon provide evidence for gamma ray attenuation due to collisions of gamma rays with low-energy photons from the MRF, thus allowing to test the model predictions in an independent way (Paper II in this series) ${ }^{6}$.

Acknowledgements. We thank Stephane Charlot for providing the latest release of the population synthesis templates (GISSEL99), and Joel Primack for communicating the SFR function from structure formation theory prior to publication. We also thank Floyd Stecker for fruitful discussions. This work is part of a research project supported by the BMBF under code DESY-HS/AM09731M45. DH acknowledges support and hospitality during visits to the Universitäts-Sternwarte Göttingen.

\section{References}

Armand, C. 1994, A\&A, 284, 12

Baugh, C. M., Frenck, C. S, \& Lacey, C. G. 1998, ApJ, 498, 504

Bernstein, R. A., Freedman, W., \& Madore, B. F. 2002, in prep.

\footnotetext{
${ }^{6}$ At http://wwww.astro.uni-wuerzburg.de/theorie/ we provide a web tool for online calculations of the MRF, allowing users to select values of key parameters.
}

Blain, A. W., \& Longair, M. S. 1993, MNRAS, 264, 509

Boissier, S., \& Prantzos, N. 1999, MNRAS, 307, 857

Bruzual, A. G., \& Charlot, S. 1993, ApJ, 405, 538

Bruzual, A. G. 2000 [astroph-0011094]

Connolly, A. J., Szalay, A. S., Dickinson, M., Subbarao, M. U. \& Brunner, R. J. 1997, ApJ, 486, L11

Dove, J. B., Shull, J. M., \& Ferrara, A. 2000, ApJ, 531, 846

Dwek, E., Arendt, R. G., Fixsen, D. J., et al. 1997, ApJ, 475, 565

Dwek, E., \& Arendt, R. G. 1998, ApJ, 508

Dwek, E., \& Hauser, M. G. [astro-ph 0105539]

Ellis, R. S., Colless, M., Broadhurst, T., Heye, J., \& Glazerbrook, K. 1996, MNRAS, 280, 235

Elbaz, D., Cesarsky, C. J., Fadda, D., et al. 1999, A\&A, 351, 37

Fields, B. 1999, ApJ, 515, 603

Fixsen, D. S., Dwek, E., Matter, J. C., Bennett, C. L., \& Shafer, R. A. 1998, ApJ, 508, 123

Finkbeiner, D. P., Davis, M., \& Schlegel, D. J. 2000, ApJ, 544, 81

Flores, Hanner, F., Thuan, T. X., et al. 1999, ApJ, 517, 148

Franceschini, A. 2000, The Extragalactic Infrared Background and its cosmological Implications, IAU Symp., 204, 283

Franceschini, A., Aussel, H., Cesarsky, C. J., Elbaz, D., \& Fadda, D. 2001, A\&A, 378, 1

Gallego, J., Zamorano, J., Aragon-Salamanca, A., \& Rego, M. 1995, ApJ, 455, L1

Giallongo, E., Fontana, A., Cristiani, S., \& D'Odorico, S. 1996, ApJ, 466, 46

Girardi, L., Bressan, A., Bertelli, G., \& Chiosi, C. 2000 A \& AS 141, 371

Gorjian, V., Wright, E. L., \& Chary, R. R. 1999, AAS, 195, 1402

Gorjian, V., Wright, E. L., \& Chary, R. R. 2000, ApJ, 536, 550 
Granato, G. L., Lacey, C. G., Silva, L., et al. 2000, ApJ, 542, 710

Guiderdoni, B., Hivon, E., Bouchet, F. R., \& Maffei, B. 1998, MNRAS, 295, 877

Hauser, M. G., Arendt, R. G., Kelsall, T., et al. 1998, ApJ, 508,25

Hauser, M. G., \& Dwek, E. 2001, ARA\&A, 39, 249

Hughes, D. H., Serjeant, S., Dunlop, J., et al. 1998, Nature, 394,241

Juvela, M., Mattila, K., \& Lemke, D. 2000, A\&A, 360, 813

Kauffmann, G., White, S. D. M., \& Giuderdoni, B. 1993, MNRAS, 264, 201

Kauffmann, G., Colberg, J. M., Diaferio, A., \& White, S. D. M. 1999, MNRAS, 303, 188

Kennicutt, R. C. 1983, ApJ, 272, 54

Kennicutt, R. C. 1998, ARA\&A, 36, 189

Kim, D.-C., Veilleux, S., \& Sanders, D. B. 1998, ApJ, 508, 627

Kim, D.-C., \& Sanders, D. B. 1998, ApJS, 119, 41

Lagache, G., Abergel, A., Boulanger, F., Désert, F. X., \& Puget, J.-L. 1999, A\&A, 344, 322

Leitherer, C., Alloin, D., Fritz-v. Alversleben, U., et al. 1996, PASP, 108, 996

Lilly, S. J., Le Fevre, O., Hammer, F., \& Crampton, D., et al. 1996, ApJ 460, L1

Loeb, A., \& Waxman, E. 2000, Nature, 405, 156

Madau, P. 1997(I), ApJ, 475, 429

Madau, P. 1997(II), sfnf. Conf, 481M

Madau, P., Pozzetti, L., \& Dickinson, M. 1998, ApJ, 498, 106

Madau, P. 1999 [astro-ph/9907268]

Madau, P. 2000 [astro-ph/0005106]

Madau, P., \& Pozzetti, L. 2000, MNRAS, 312, L9

Malkan, M., \& Stecker, F. W. 1998, ApJ, 496, 13

Malkan, M., \& Stecker, F. W. 2001, ApJ, 555, 641

Mushotsky, R. F., Cowie, L. L., Barger, A. J., \& Arnaud, K. A. 2000, Nature, 404, 459

Nagamine, K., Cen, R., \& Ostriker, J. P. 2000, ApJ, 541, 25

Osterbrook, D. E. 1998, University Science Books Astrophysics of Gaseous Nebulae and AGN

Peebles, P. J. E. 1993, Principles of Physical Cosmology (Princeton University Press)

Puget, J. L., Lagache, G., Clement, D. L., et al. 1999, A\&A, 345,29
Puget, J. L., \& Lagache, G. 2000, The Extragalactic Infrared Background and its cosmological Implications, IAU Symp., 204, 233

Pei, Y. C., \& Fall, S. M. 1995, ApJ, 454, 69

Pei, Y. C., Fall, S. M., \& Hauser, M. G. 1998, A\&AS, 193, 4906

Pei, Y. C., Fall, S. M., \& Hauser, M. G. 1999, ApJ, 522, 604

Pettini, M., Steidel, C. C., Adelberger, K. L., et al. 1998, ASP Conf. Ser., 148, 67

Prantzos, N., \& Silk, J. 1998, ApJ, 507, 229

Primack, J. R., Bullock, J. S., Somerville, R. S., \& MacMinn, D. 1999, APh, 11, 93

Pozzetti, L., Madau, P., Zamorani, G., Fergusson, H. C., \& Bruzual, A. C. 1998, MNRAS, 298, 1133

Renault, C., Barrani, A., Lagache, G., \& Puget, J.-L., et al. 2001, A\&A, 371, 771

Rowan-Robinson, M. 2001, ApJ, 549, 745

Ruiz-Lapuente, P., Casse, M., \& Vangioni-Flam, E. 2000, ApJ, 549, 483

Salamon, M. H., \& Stecker, F. W. 1998, ApJ, 439, 547

Sauvage, M. 1997, The Interstellar Medium in Galaxies, Astrophys. and Space Sci. Library, vol. 219, ed. J. M. von der Hulst (Kluwer Academic Publisher)

Soifer, B. T., Sanders, D. B., Madore, B. F., et al. 1987, ApJ, 320,238

Somerville, R., \& Primack, J. R. 1999, MNRAS, 310, 1087

Spinoglio, L., Malkan, M. A., Rush, B., Carrasco, L., \& Recillas-Cruz, E. 1995, ApJ, 453, 616

Stanev, C. C., \& Franceschini, A. 1998, ApJ, 494, 159

Steidel, C. C., Adelberger, K. L., Giavalisco, M., Dickinson, M., \& Pettini, M. 1999, ApJ, 519, 1

Sreekumar, P. 2000, in Proc. of the 5th Compton Symp., ed. M. L. McConnell, \& J. M. Ryan (AIP: N.Y.), 510, 459

Tan, J. C., Silk, J., \& Balland, C. 1999, ApJ, 522, 579

Vladilo, G., Bonifacio, P., Centurion, M., \& Molaro, P. 2000, ApJ, 543, 24

Valageas, P., \& Silk, J. 1999, A\&A, 347, 1

Watanabe, K., Hartmann, D. H., Leising, M. D., \& The, L.-S. 1999, ApJ, 516, 285

Weidenspointner, G. 1999, Ph.D. Thesis, Technische Universität München

White, S. D. M., \& Frenk, C. S. 1991, ApJ, 379, 52 